\title{
Research and Application of Big Data Technology in Mobile Communication Billing
}

\author{
Mr. Hong Yunguo \\ Dalian Vocational and Technical College, Post Code 116035
}

Key words: big data; mobile communication; billing area; application

\begin{abstract}
In the current era of big data era, the rapid integration of information and data has been realized in the development of mobile Internet technology. The potential for mobile communications has also been tapped. With the increasing popularization and expansion of mobile terminal technologies, there are more users of mobile terminals. There are also more communication and accounting bills to be processed in the system, and the technologies need to be processed with advanced technologies. This article will analyze the application of big data technology in the field of mobile communication billing and put forward corresponding measures.
\end{abstract}

\section{Introduction}

In the current rapid development of information technology, the widespread application and popularity of mobile terminal technology, the number of mobile terminal technology is also increasing, the system needs to handle more bills, which poses a great challenge to the system's processing power. The existing technologies in the traditional system can no longer meet the current application status and need to constantly explore a new data processing method to handle all the information on mobile communications. Big data has become a new technology that has been widely used in recent years. It has been applied for various industries to realize effective data processing and analyze and to adapt to the current trend of social development. Big data mainly uses a kind of computer software to realize the collection, collation and collection of data. It is applied to the advanced computer network technology to deal with a large amount of data and information, and can obtain valuable information resources from it. It has become the core technology of the current Internet.

\section{Research Background}

As an advanced means of information technology, the development and application of big data is also a trend to adapt to the development of social information technology. With the advent of the information age and the increasing amount of information, the traditional data storage and analysis technology can no longer adapt to the current new In this case, the application of big data technology will become a new trend. It can be said that cloud computing is the technology foundation of big data. Big data provides value for cloud computing, such as virtualization and distribution storage technology and a large number of data management information for the processing of big data and laid a good foundation for providing the appropriate level of technical support. The characteristics of big data technology are: large amount of computing and storage; diversity characteristics; analysis and processing speed, etc., but also need to pay attention to the safety of data and information in the process of data processing, which also is a role focus the calculation and storage, need to analyze and summarize in the actual business work, pay attention to the actual application function.

Mobile communication enterprises are a pillar enterprise in our country. There are tens of thousands of users in our country who use mobile terminals. In the process of communication, there will be a large number of billing bills, and as the number of mobile services increases, the data processing is also increasing. The traditional data processing functions cannot meet the current development needs, but also provide the corresponding opportunity for the application of big data technology. 


\section{Related Content of Mobile Communication Billing}

Mobile communication billing is the core of the work of mobile communications companies, it will be related to the effective development of all business operations, the stable operation of billing system are an important basis of the development of mobile communications companies. The current use of mobile communications is also becoming more, but also opened a wide range of business content, including billing business projects is more needed to deal with the bill is also more, for a variety of data processing, need a relevant software to deal with. In the field of mobile communications, there are a lot of contents that need to be charged, including communication charging to local users, roaming charging with inter-provincial users and current application IoT charging etc. These various charging items are needed to have the corresponding billing system for processing, through the collection of a large number of bill information, unified processing. At present, with the continuous expansion of the service of the mobile terminal, a large number of charging bills bring great challenges to the traditional processing system. The conventional processing system needs to be optimized, upgraded and improved constantly. For system upgrades often cost a lot of money, but the functionality is limited.

\subsection{Mobile Communication System Module Analysis}

Relevant tariff verification, the verification of mobile communications charges, need to test each dialogue, including the format of the dialogue is consistent with the relevant business requirements, if you do not meet the requirements of the corresponding error code, the situation needs to be returned for the error [1]. Also need to test whether the relevant costs recorded in the bill meet certain tariffs. The check and retrieval of the bill of lading requires comparative analysis of the current bill contents and the historical bill contents. The single test needs to scan the massive bill stored in the database, import the bill through the detected database into the corresponding database, although the import process is relatively simple, but the amount of data is relatively large, it will also bring great challenges to the processing [2].

\subsection{Current Processing System Applications}

In the current rapid growth of mobile users and services, the system needs to handle more bills, and in some holidays, the bill is a sharp increase in the number of existing software systems are no longer able to support the conversation costs single processing, and a variety of new business single volume is also the data processing functions require more higher [3]. Therefore, we need to introduce new business processes software and systems, using cloud computing and big data technology for processing, can solve the existing problems. And cloud computing and big data technology has been widely used in many industries, each industry need to develop their own strategic programs in accordance with the development of their own business, cloud computing and big data technology will be applied to them, the most widely used in mobile communications is " Great cloud " technology, which is also a relatively successful technology in China's current mobile [4].

\subsection{Application of Hadoop in Mobile Communications}

Hadoop is a distributed computing system that processes large data using a simple computer model. It belongs to a collection of projects that mainly include distributed file systems and common tools as well as distributed databases and big data analytics platforms. One of the file system and distributed computing framework as the core part of data processing for other modules to lay the foundation for the application of some of the system is responsible for managing the file system naming and management of client access to the file and the other part is managing their own file storage and related data information interaction [5]. For example, mapreduce is a software framework that can be applied to large-scale dataset processing operations and belongs to a programming model that can handle very large data sets. In the field of mobile communication billing system data processing, through the use of distributed data processing, which can effectively improve the data performance of the system runtime, the kind of software technology can 
effectively improve the data high-speed random read and write support which can be increased by adding nodes. The way to expand the corresponding data analysis, which can achieve flexibility and economy in data processing [6].

In the current data size, the system's processing power and scalability are faced with major problems, it will also affect the stable operation of the system, but also in the peak of the business phase of the system processing functions may be paralyzed from this situation, the system problems mainly read and write functions in the processing of database, the amount of data processed is too large, and the processing time limit is specified, the use of software optimization or hardware upgrade are able to meet the short-term needs, in the long-term system application process, there is still a big flaw [7]. Considering all kinds of comprehensive factors, by introducing distributed computer processing system to replace the original processing database, a large amount of data and information can be placed in the cluster in the distributed system, some of the more complex result data in the relational database, it can effectively achieve unified settlement and statistical inquiry [8]. For example, bill-to-bill and result-to-bill data from all provinces are distributed to four different distributed data clusters, each of which will have fixed data access requirements concurrently to reflect the scalability and advantages of the system.

\section{Conclusion}

To sum up, in the current large-scale application of mobile communications, the data processing in the field of communication charging is facing a great problem. In the face of a large amount of data, how to choose a suitable data processing technology is also a problem currently to be fully considered through the introduction of advanced distributed data processing system, it can realize the processing of massive data, ensure the stability and security of the whole system operation and lay a solid foundation for the scalability of the system.

\section{References}

[1] Wu Junling.Application of big data analysis in mobile communication network optimization [J]. GANSU, 2017,46 (09): 7-9 + 84 .

[2] Jin Ziyang.Application and development strategy of big data in mobile communication network [J].New China New Telecommunications, 2016,18 (10): 64.

[3] Dang Yongliang.Application of big data analysis in mobile communication network optimization [D]. Central China Normal University, 2015.

[4] Liu Sheng. Application of Big Data Technology in Mobile Communication Billing [J].Mobile Communications, 2013,37 (15): 41-46.

[5] Li Cheng-Qi, Lei Chun, Wang Rui-Shen, ZHOU Xiao-Le.Research and Implementation of GPRS Call Scheduling Scheme in Mobile Communication Charging System [J]. Mobile Communications, 2016,40 (14): 76-79

[6] Liu Dan.Application of big data in mobile communication [J]. Inner Mongolia Coal Economy, 2016 (12): $34+115$.

[7] Liu Sheng. Application of Big Data Technology in Billing of Mobile Communication [J].Mobile Communications, 2013,37 (15): 41-46.

[8] Gao Mingming, Hui Xiaowei, Lin Sen. Discussion on the method of charging for mobile communication [J].Journal of Liaoning Technical University, 2008 (S1): 219-221. 